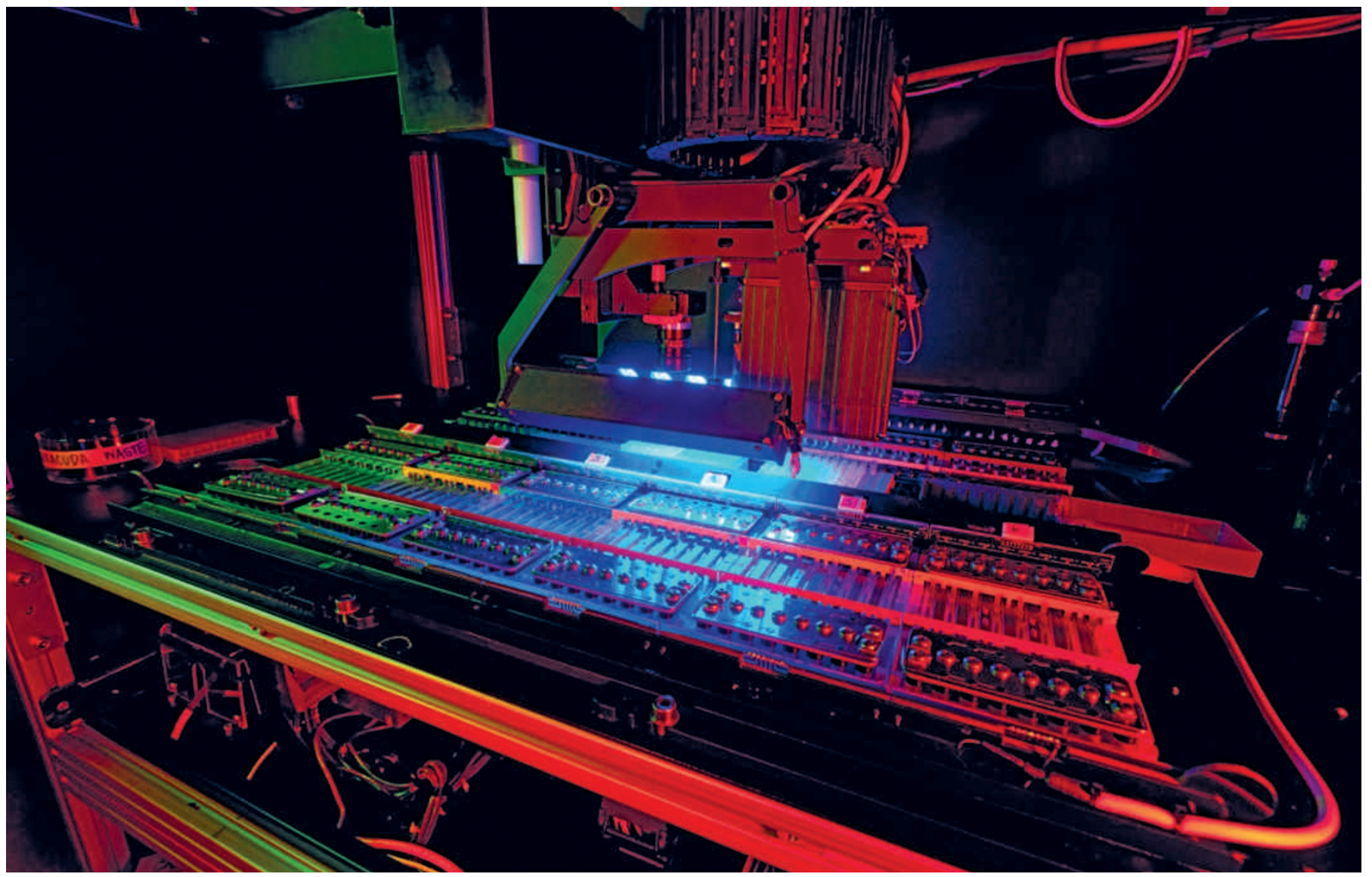

The use of genome sequencers has allowed researchers to identify many targets for possible schizophrenia drugs.

GENETICS

\title{
Unravelling complexity
}

\section{A massive research collaboration is revealing hundreds of genes underlying schizophrenia risk, and may point the way to targeted treatments.}

\section{BY JESSICA WRIGHT}

A plot of the genes associated with a psychiatric disease isn't the kind of image you'd normally find on a T-shirt. But at the 2013 Symposium on Emerging Genetics and Neurobiology of Severe Mental Illness, held in September at the Broad Institute in Cambridge, Massachusetts, more than 250 attendees snatched up garments displaying just such an image, along with the catchphrase "Schizophrenia genetics - a reality at last!" It's a clear sign that schizophrenia researchers are excited about recent advances in pinpointing the genetic causes of the disease.

Seven years ago, they had all but given up on this type of genetic analysis, which links single nucleotide changes in a gene with the likelihood of developing a disorder. Genomewide association studies (GWAS) were failing to identify schizophrenia genes with statistical certainty. But in the past two years, researchers have identified more than 100 genome-wide significant hits, implicating almost 700 genes. That tally may soon grow into the thousands. This remarkable progress has rekindled hope that researchers may be able to use genetics to identify targets for schizophrenia drugs.

Studies on twins have shown that as much as $80 \%$ of the risk of developing schizophrenia comes from the genes. But pinpointing targets for drug developers has proved an elusive goal. As a result, most schizophrenia drugs treat only the overt symptoms - the imaginary voices and other elements of psychosis - and do little to address the broad effects on the brain. They also have severe side effects. "Pharmaceutical companies have almost given up on the central nervous system entirely," says Steven Hyman, director of the Broad Institute's Stanley Center for Psychiatric Research. But now, he says, this may be about to change.
Much of the progress comes from technologies that allow researchers to scan large swaths of the genome to reveal unexpected genetic links, instead of looking only at individual genes. In addition, schizophrenia researchers have turned to the methods of 'big science', banding together to generate statistical power.

The driver behind this effort is the Psychiatric Genomics Consortium (PGC), which pools genetic data from researchers at 60 institutions in 19 countries. The PGC is collecting genomes focusing on five psychiatric disorders, including autism and attention deficit hyperactivity disorder (ADHD). But the number of genomes collected for schizophrenia surpasses the others: nearly 40,000 at a recent count.

The high level of collaboration for schizophrenia reflects the fact that most participants know how hard it is to unravel this complexity alone, says Patrick Sullivan, a geneticist at the University of North Carolina, Chapel Hill, and 
the PGC's lead investigator. "We had to come together as a field."

The early findings reveal that schizophrenia results from mutations in multiple genes working together in different combinations. As researchers unearth more gene candidates, they are discovering biological pathways that may serve as entry points for drug therapies. "The scale of the polygenicity is surprising," says Steven McCarroll, a geneticist at Harvard Medical School in Boston, Massachusetts. "But the exciting thing is that these genetic hits aren't just random hits on the dartboard of the genome. They are aggregating in specific molecular complexes and pathways."

\section{COMMON AND RARE}

The first hits identified in schizophrenia were so-called rare variants - genetic mutations that usually have strong effects on their corresponding proteins. These mutations, which nearly always lead to symptoms and are fairly easy to identify, are often found in an affected individual but not in their unaffected parents or siblings. Studies suggest that rare variants account for up to $20 \%$ of schizophrenia cases. The remaining genetic hits are likely to be common variants, which are found throughout the population and have relatively mild effects, but in combination may be sufficient to tip the scales towards schizophrenia. Individuals may also have a combination of both variants.

It makes sense that variants with mild effects may be important, as many are in genes that influence crucial pathways, such as the signalling junctions between neurons. But schizophrenia is not characterized by the severe intellectual disability and epilepsy that is a hallmark of disrupted brain pathways. In fact, people with schizophrenia tend not to develop problems until adolescence. "The synapse is a hugely complicated machine - how can we mess with it in such a way that we can get a disorder where people develop normally for a couple of decades?" asks Sullivan.

Until recently, researchers didn't have the statistical power needed to test common variants' influence in schizophrenia. But the sheer number of participants gathered through the PGC allows researchers to pinpoint individual variants that contribute to schizophrenia risk.

In a 2013 Nature Genetics study, Stephan Ripke, a geneticist at the Broad Institute, and colleagues analysed the genomes of more than 21,000 individuals with schizophrenia ${ }^{1}$. They identified 22 variants associated with schizophrenia at the level of genome-wide significance. At the Broad conference, Ripke presented - and wore - the latest data from this project: an analysis of 35,000 people with schizophrenia and 47,000 controls that has yielded more than 100 statistically significant hits implicating 672 genes. In the next year, the consortium aims to have analysed 100,000 genomes.

One of the new hits is in the gene DRD2, which makes a receptor for dopamine, a chemical messenger that helps mediate reward. The discovery is not surprising, as all the existing drug therapies for schizophrenia target the dopamine pathway. But it is a proof of principle that the PGC can identify useful drug targets. $D R D 2$ is a "home run example", says Ripke. "It is very valid to assume that among the other hits there are other drug targets."

Common variants are present throughout the population, so researchers suspect they work in combination with environmental factors. "It may be that neuronal function is perfectly fine under most circumstances, but if a body of neurons gets stressed in a certain way that can tip a person over," says Sullivan.

To discern the environmental contribution to schizophrenia's genetic risk, the PGC is collaborating with epidemiologists Preben Bo Mortensen at Aarhus University in Denmark and John McGrath at the University of Queensland in Australia. Mortensen has access to genetic information from 2.2 million people born in Denmark since 1981 who had blood collected at birth. His group aims to develop a polygenic risk score for 85,000 of them, predicting each individual's likelihood of developing schizophrenia from their composition of common variants. The researchers will also survey other factors linked to schizophrenia risk, such as low birth weight or low vitamin D levels, enabling them to tease apart the genetic and environmental contributions to schizophrenia.

\section{OPENING THE EXOME}

Despite the explosion of knowledge about common variants, some researchers continue to see the most promise in rare mutations. These mutations can reveal pathways that are undeniably implicated in the disorder, says David Goldstein, a geneticist at Duke University in Durham, North Carolina. "The part of the genetic architecture I'm interested in is where a smaller set of higher-impact mutations imply risk that I can study biologically and use as guidance for drug development," he says.

To search for rare variants that remove, insert or change single DNA nucleotides, researchers are sequencing exomes, the protein-coding regions of the genome. Today's exome studies still have too few sequences to reveal a statistically significant role for a particular gene. But they do implicate certain pathways, such as the development and migration of new neurons and signalling through NMDA ( $N$-methyl-Daspartate) receptors. The studies also suggest an important role for proteins at the receiving ends of synapses (the postsynaptic density).

The two largest exome-sequencing studies in schizophrenia so far were published in Nature in January 2014. In one, researchers sequenced the exomes of 623 people with schizophrenia and their unaffected parents ${ }^{2}$. The other looked at the exomes of 2,546 genes associated with schizophrenia or other psychiatric disorders in more than 5,000 people, divided between those with schizophrenia and controls ${ }^{3}$. Both studies found several hits in genes regulated by the fragile X mental retardation protein, which is missing in fragile $\mathrm{X}$ syndrome, a disorder that can lead to intellectual disability and autism.

Rare spontaneous mutations may also take the form of copy number variants (CNVs) - duplications or deletions of stretches of a chromosome. Roughly one in 20 people with schizophrenia have CNVs of more than 100 kilobases ${ }^{4}$. Each CNV spans a number of genes, one or many of which may be associated with the disorder. Those implicated in schizophrenia tend to encompass genes that also play a role in the postsynaptic density and NMDA signalling.

The most well known of these CNVs, a 3-megabase deletion in the 22q11.2 chromosomal region, leads to schizophrenia or psychosis about $25 \%$ of the time. One recent study suggests that duplication of this region may protect against schizophrenia while increasing the risk of autism ${ }^{5}$, demonstrating the complex and overlapping relationships in the genetics of psychiatric disorders.

Indeed, different genetic analyses are highlighting some of the same genes and pathways. Both the PGC GWAS and the recent exome studies, for example, identified multiple hits for genes involved in the L-type calcium channel, which controls muscle and cardiac activity. The multiple hits in these calcium-channel subunits, using different genetic analyses, suggest that these may be the most promising potential therapeutic targets discovered so far.

Researchers hope to find more targets as they identify further gene candidates. To push their statistical power even higher, a team at PGC designed the Psych Chip - a device that makes it cheaper to screen the genomes of a large number of individuals (roughly US\$50 per person). The chip covers 60,000 common and rare variants that were implicated in schizophrenia in previous studies but fell short of statistical significance. It also covers genes within CNVs. The researchers aim to test 100,000 individuals with psychiatric disorders in the next year. Based on results so far, this analysis should generate at least four more statistically significant hits for every 1,000 individuals, says Ripke.

Two years from now, researchers will need a larger T-shirt to flaunt their findings. "It's becoming clear that we are far from the end of the discovery step," says Ripke. "A hundred hits will not be the answer. There will be many hundreds if not thousands, which frightens many people. But we are definitely not slowing down."

Jessica Wright is a freelance science writer based in New York City.

1. Ripke, S. et al. Nature Genet. 45, 1150-1159 (2013)

2. Fromer, M. et al. Nature 506, 179-184 (2014)

3. Purcell, S. M. et al. Nature 506, 185-190 (2014).

4. Kirov, G. et al. Mol. Psychiatry 17, 142-153 (2012).

5. Rees, E. et al. Mol. Psychiatry 19, 37-40 (2014). 